\title{
The Concise Health Risk Tracking-Self Report: Psychometrics within a placebo-controlled antidepressant trial among depressed outpatients
}

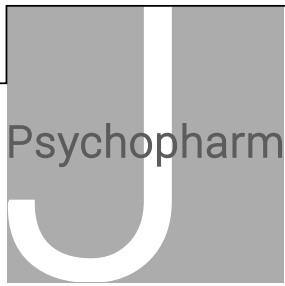

Journal of Psychopharmacology 2019, Vol. 33(2) 185-193 (c) The Author(s) 2019 Article reuse guidelines: sagepub.com/journals-permissions DOI: $10.1177 / 0269881118817156$ journals.sagepub.com/home/jop

\author{
Joseph M Trombello', Michael 0 Killian ${ }^{2}$ (iD, \\ Bruce D Grannemann ${ }^{1}$, Augustus John Rush ${ }^{3-5}$, Taryn L Mayes ${ }^{1}$, \\ Ramin V Parsey ${ }^{6}$, Melvin McInnis ${ }^{7}$, Manish K Jha ${ }^{1}$, Aasia Ali ${ }^{1}$, \\ Patrick J McGrath ${ }^{8,9}$, Phil Adams ${ }^{9}$, Maria A Oquendo ${ }^{10}$, \\ Myrna M Weissman ${ }^{8,9}$, Thomas J Carmody ${ }^{1}$ and Madhukar H Trivedi ${ }^{1}$
} (\$)SAGE

\begin{abstract}
Background/aims: While substantial prior research has evaluated the psychometric properties of the 12-item Concise Health Risk Tracking-Self Report $\left(\mathrm{CHRT}_{-} \mathrm{SR}_{12}\right)$, a measure of suicide propensity and suicidal thoughts, no prior research has investigated its factor structure, sensitivity to change over time, and other psychometric properties in a placebo-controlled trial of antidepressant medication, nor determined whether symptoms change throughout treatment.

Methods: Participants in the multi-site Establishing Moderators and Biosignatures of Antidepressant Response in Clinical Care (EMBARC) study ( $n=278$ ) provided data to evaluate the factor structure and sensitivity to change over time of the CHRT-SR ${ }_{12}$ through eight weeks of a clinical trial in which participants received either placebo or antidepressant medication (sertraline).

Results/Outcomes: Factor analysis confirmed two factors: propensity (comprised of first-order factors including pessimism, helplessness, social support, and despair) and suicidal thoughts. Internal consistency ( $\alpha$ 's ranged from 0.69-0.92) and external validity were both acceptable, with the total score and propensity factor scores significantly correlated with total scores and single-item suicidal-thoughts scores on the self-report Quick Inventory of Depressive Symptoms and the clinician-rated 17-item Hamilton Rating Scale for Depression. Through analyzing CHRT-SR ${ }_{12}$ changes over eight treatment weeks, the total score and both the factors decreased regardless of baseline suicidal thoughts. Change in clinician-rated suicidal thoughts was reflected by change in both the total score and propensity factor score.

Conclusions/interpretation: These results confirm the reliability, validity, and applicability of the CHRT-SR ${ }_{12}$ to a placebo-controlled clinical trial of depressed outpatients receiving antidepressant medication.
\end{abstract}

Keywords

Concise Health Risk Tracking-Self Report, psychometrics, major depression, suicidal thoughts, suicide ratings

\section{Introduction}

Suicide represents a major public health concern, being the $10^{\text {th }}$ leading cause of death and costing over US\$50 billion annually in the USA (CDC, 2016). Suicide is also a major problem globally: with almost 800,000 people worldwide committing suicide each year, suicide is the second leading cause of death worldwide among individuals aged $15-29$ years, and 78\% of global suicides occur in low and middle-income countries (WHO, 2018). Suicidal ideation is one of the nine symptoms of major depressive disorder (MDD), and the association between this disorder, suicidal ideation, and attempts has been well-documented (Kessler et al., 1999; Mann et al., 2005). A commonly used measure of suicidal ideation and attempts presently, the clinician-rated Columbia-Suicide Severity Rating Scale (C-SSRS; Posner et al., 2011), is comprehensive, and in research settings has been shown to predict suicide attempts (Posner et al., 2011). It can, however, be burdensome, requiring significant training to administer (Reilly-Harrington et al., 2016). The C-SSRS has been criticized
${ }^{1}$ Department of Psychiatry, University of Texas Southwestern Medical Center, Dallas, TX, USA

${ }^{2}$ College of Social Work, Florida State University, Tallahassee, FL, USA

${ }^{3}$ Department of Psychiatry, Duke Medical School, Durham, NC, USA ${ }^{4}$ Department of Psychiatry, Texas Tech University Health Sciences Center, Permian Basin, TX, USA

${ }^{5}$ Duke-National University of Singapore, Singapore

${ }^{6}$ Department of Psychiatry, Stony Brook University, Stony Brook, NY USA ${ }^{7}$ Department of Psychiatry, University of Michigan, Ann Arbor, MI USA ${ }^{8}$ Department of Psychiatry, Columbia University, New York, NY USA ${ }^{9} \mathrm{New}$ York State Psychiatric Institute and Department of Psychiatry, College of Physicians and Surgeons of Columbia University, New York, NY USA ${ }^{10}$ Department of Psychiatry, University of Pennsylvania, Philadelphia, PA, USA

Corresponding author:

Madhukar Trivedi, Center for Depression Research and Clinical Care, UT Southwestern Medical Center, 5323 Harry Hines Boulevard, Dallas, TX 75390-9119, USA.

Email: madhukar.trivedi@utsouthwestern.edu 
by researchers advocating a competing suicide measure for its conceptual and psychometric limitations and the propensity for raters to misclassify participant responses into the incorrect category (Giddens et al., 2014). However, the C-SSRS has also been shown to be equivalent to the Suicide Tracking Scale and the Sheehan Suicidality Tracking Scale in classifying suicidal ideation and behavior in general (Youngstrom et al., 2015). Nonetheless, there is value in examining simpler, self-report measures of suicidal ideation that also tap into risk factors for suicide attempts, such as interpersonal estrangement and perceived lack of social support (Joiner, 2005), and negative thinking, such as helplessness/hopelessness (Abramson et al., 1989, 2000).

The Concise Health Risk Tracking-Self Report (CHRT-SR) scale (Trivedi et al., 2011) is a well-established measure of suicidal risk and ideation. Questions assess for not only suicidal thoughts but also pessimism, helplessness, perceived lack of social support, and despair. Samples with both bipolar (Ostacher et al., 2015; Reilly-Harrington et al., 2016; Villegas et al., 2018) and unipolar depressed participants (Morris et al., 2010; Trivedi et al., 2011; Villegas et al., 2018) suggest strong reliability and validity, as well as sensitivity to change throughout antidepressant treatment (de la Garza et al., 2018). Baseline suicidal thoughts as assessed through the CHRT-SR are also associated with worse antidepressant treatment outcomes (Zisook et al., 2011). Latent class analysis has recently identified four groupings of participants based upon changes in CHRT-SR suicidal ideation over six months of treatment for bipolar depression (Kohler-Forsberg et al., 2017). As well, treatment research has indicated that various interventions, including computerized software to recommend medication treatments (Iosifescu et al., 2016), L-methylfolate (Nierenberg et al., 2017), and cognitive therapy (Celano et al., 2017) are associated with reductions in suicidal ideation as assessed by the CHRT-SR. Finally, the CHRT-SR has also been employed in other psychiatric disorders, including cannabis use disorder (Foster et al., 2016), stimulant use disorder (Sanchez et al., 2018), and depressed adolescents in an intensive outpatient program for suicidality (Mayes et al., 2018). In spite of its extensive research backgroud, no prior research has examined the reliability, validity, factor structure and sentisivity to change of the CHRT-SR in placeo-controlled antidepressant clinical trial, yet the measure is likely to be used in these critical studies.

This report aims at replicating and extending prior research on the factor structure of the 12-item CHRT-SR (CHRT-SR ${ }_{12}$ ) and documenting its sensitivity to change over eight weeks of acute treatment in a sample of depressed outpatients receiving either placebo or active antidepressant medication (sertraline). It is the first report, to our knowledge, to evaluate the psychometric properties of the CHRT in a placebo-controlled antidepressant study, a critical step in evaluating the measure's performance.

\section{Method}

\section{Study design and participants}

The study design was reviewed and approved by each site's Institutional Review Board (IRB), and, before enrollment, all participants signed written informed consent.
Participants for the multi-site Establishing Moderators and Biosignatures of Antidepressant Response in Clinical Care (EMBARC) study were recruited from July 2011-December 2015 through direct referrals from physicians, flyers, and advertising. The study included 296 participants aged 18-65 years with recurrent or chronic single episode MDD or dysthymia, with the first onset before age of 30 years. Additional exclusion criteria included inadequate response or poor tolerability to study medications; a failed antidepressant trial of sufficient dosage and duration within the current episode; a history of psychotic or bipolar disorder; and substance dependence (except for nicotine) within the past six months or substance abuse within the past two months. Seventeen participants were excluded who dropped out before taking study medication or providing any post-baseline data, and one participant was excluded because of missing CHRT-SR $_{12}$ data, making the sample $n=278$. There were four sites for data collection: University of Texas (UT) Southwestern Medical Center, Columbia University, Massachusetts General Hospital, and the University of Michigan.

The EMBARC study entailed two phases: an eight-week placebo-controlled trial and a subsequent eight weeks in which nonresponders to placebo were switched to an active medication, sertraline, to determine biological, physiological, cognitive, and genetic biomarkers of response to sertraline and bupropion (Trivedi et al., 2016). Sertraline is a selective serotonin reuptake inhibitor (SSRI) that reduces depressive symptoms by preventing the reuptake of serotonin, thus allowing more of the neurotransmitter. In the first phase, subjects were randomized into a placebo-controlled, double-blind trial of sertraline as compared to placebo, which was designed to examine biomarkers for depression treatment selection and effect.

Participants completed a baseline session that included a number of clinician-administered and self-report questionnaires, including the CHRT-SR ${ }_{12}$, as well as electroencephalogram and magnetic resonance imaging tasks (Trivedi et al., 2016). At baseline, participants were randomized to receive either placebo or sertraline and followed during laboratory visits at weeks 1, 2, 3, 4, 6 , and 8 . This report analyzes the first eight weeks of study enrollment. More comprehensive information about the EMBARC study is available (Trivedi et al., 2016).

\section{Measures}

The CHRT-SR 12 scale (Trivedi et al., 2011) is comprised of two factors: propensity and suicidal thoughts. The propensity factor includes nine items that tap into four separate domains: pessimism, helplessness, perceived lack of social support, and despair. The suicidal thoughts factor is comprised of three separate items (items 10-12) that assess for thoughts and plans of killing oneself. All items are scored on a five-point Likert scale with responses ranging from "strongly disagree" (zero) to "strongly agree" (four), thereby creating a total score that ranges from $0-48$, with higher scores showing higher levels of suicidal propensity and suicidal thoughts.

Other measures were examined for purposes of establishing validity. Specifically, these included the self-report 16-item Quick Inventory of Depressive Symptoms (QIDS-SR 16 ; Rush et al., 2003), and the clinician-assessed 17-item Hamilton Rating Scale for Depression (HAM- $\mathrm{D}_{17}$; Hamilton, 1960), using the conventional Structured Interview Guide for the Hamilton 
Depression Rating Scale (SIGH-D; Williams, 1988) and in conjunction with didactic and experiential training through rating mock videotapes. Raters were primarily at the Doctor of Medicine or Doctor of Philosophy level, but also included Bachelor's level Registered Nurses. We analyzed both the total scores from each of these two measures, as well as the single suicidal ideation (SI) item from each of these two measures, for purposes of establishing construct validity, and to determine if there were differences in associations between the self-report CHRT and both selfreport and clinician-administered measures of depressive symptoms, including suicidal thoughts. The SIGH-D specified five possible answers for suicidal ideation ( 0 - absent; 1 - feels life is not worth living; 2 - wishes (s)he were dead or any thoughts of possible death to self; 3 - suicidal ideas or gestures; 4 - attempts at suicide) while the SI item from the self-report QIDS included four possibilities $(0-$ I do not think of suicide or death; 1 - I feel that life is empty or wonder if it's worth living; 2 - I think of suicide or death several times a week for several minutes, 3 - I think of suicide or death several times a day in some detail, or I have made specific plans for suicide or have actually tried to take my life).

\section{Data analysis}

Confirmatory factor analysis (CFA) was used to assess the fit of the CHRT-SR 12 model to the EMBARC study data. As in previous evaluations of the psychometric properties of the CHRT-SR ${ }_{12}$, propensity was included as a second-order factor composed of the four first-order factors (i.e. pessimism, helplessness, social support, and despair). Model fit was assessed using the model chi-square value ( $\chi^{2}$; Kline, 2016), model chi-square per degrees of freedom ( $\chi^{2} / \mathrm{df}$; Bollen, 1989), root mean square error of approximation (RMSEA; Kline, 2016) including the $90 \%$ confidence interval (CI), Bentler comparative fit index (CFI; Hu and Bentler, 1999) and Tucker-Lewis index (TLI; Kline, 2016), and standardized root-mean-square residual (SRMR; Brown, 2006). CFA modeling was conducted in the Mplus software package, version 8.0, using full-information maximum likelihood information estimation. Internal consistency reliability was obtained for each CHRT-SR 12 factor, and the overall CHRT-SR 12 total score. Construct validity of the CHRT-SR ${ }_{12}$ scores were tested through their association (Spearman's rho rank order correlations, $\rho$ ) with responses from the total score on the clinician-rated SIGH-D for the HAM-D ${ }_{17}$ and the self-report QIDS-SR ${ }_{16}$ measures, as well as for the single-item on suicidal ideation from both measures. Analyses examining the reliability and psychometric validity of the CHRT-SR ${ }_{12}$ were conducted using SPSS 25.0.

Two sets of mixed model analyses were conducted to evaluate the sensitivity of the CHRT-SR 12 total and factor scores' change over time compared to baseline clinical assessment on the HAM$\mathrm{D}_{17}$. Three groups were formed using the response from the baseline HAM-D ${ }_{17}$ single item on suicidal ideation: (a) none $(n=142)$, defined as a HAM-D $\mathrm{D}_{17}$ suicide item score of zero or "absent"; (b) one $(n=59)$, defined as HAM-D ${ }_{17}$ suicide item score of 1 or "feels life not worth living"; and (c) two/three $(n=77)$, defined as HAM$\mathrm{D}_{17}$ suicide item scores of two "wishes $\mathrm{s}(\mathrm{he})$ were dead or any thoughts of possible death to self" or three "suicidal ideas or gestures". There were no scores of four "attempts at suicide" on this item. Suicide change groups were defined as change in the HAM$D_{17}$ suicide item score between baseline and exit, or their last available data point (as 194 out of 278 participants had data at week 8 , indicating that 86 participants dropped out before week $8)$ : "decreased" $(n=100)$, "no change" $(n=159)$, and "increased" $(n=19)$. The relationship between these clinician assessments and change in the CHRT-SR ${ }_{12}$ over the course of treatment were analyzed using mixed model analyses with a main effect for group, a main effect for time, and a group $\times$ time interaction.

\section{Results}

Table 1 includes descriptive and demographic information for the 278 participants, including baseline CHRT-SR ${ }_{12}, \mathrm{HAM}-\mathrm{D}_{17}$, and QIDS-SR $_{16}$ scores, and the single SI item on HAM-D ${ }_{17}$ and QIDS-SR $_{16}$.

\section{Factor validity}

Factor analysis of the CHRT-SR 12 supported the fit of the model to the EMBARC data (Figure 1). Though $\chi^{2}$ model value was significant $\left(\chi^{2}=133.16, \mathrm{df}=49, p<0.001\right)$, the $\chi^{2} / \mathrm{df}$ value of 2.68 was below the accepted threshold of three and indicated model fit. The RMSEA value of 0.079 (90\% CI of $0.063-0.095)$, was less than 0.08 and demonstrated model fit. The CFI and TLI tests compared the specified model to a baseline or null model, and each offered modest support for the fit of the CHRT-SR ${ }_{12}$ model (CFI=0.923, TLI=0.897). Lastly, SRMR values less than 0.10 generally indicate model fit, which was achieved for the $\mathrm{CHRT}_{-\mathrm{SR}_{12}}(\mathrm{SRMR}=0.086)$. All factor loadings and loadings for the second-order propensity factor were statistically significant $(p<0.05)$. The propensity factor score was statistically but modestly related to the suicidal thoughts factor $(r=0.27, p<0.001)$. This indicates overlap between the two subscales but also that each is measuring unique variance associated with suicidal risk (as measured by the total score).

Table 2 shows the correlation coefficients between CHRT-SR 12 factors and constituent items. Spearman's $\rho$ correlations among the CHRT-SR ${ }_{12}$ first and second-order factors also identified in the original publication (Trivedi et al., 2011) ranged from weak to strong $(\rho=0.166-0.944$, all $p<0.01)$. Suicidal thoughts was the one factor with overall weaker correlations with other CHRT-SR 12 factors $(\rho=0.166-0.311)$, though all at a level of statistical significance (all $p<0.01$ ). All items were moderately to strongly correlated with their respective factors and overall CHRT-SR 12 total scores $(\rho=0.408-0.934$, all $p<0.001)$.

\section{Internal consistency}

Internal consistency coefficients for most of the CHRT-SR ${ }_{12}$ factors at baseline were acceptable to good at both baseline and exit (Table 3$)$. However, the reliability scores for the social support $(\alpha=0.670)$ and suicidal thoughts factors $(\alpha=0.570)$ demonstrated lower reliability coefficients. CHRT-SR 12 propensity $(\alpha=0.803)$ and total scale scores $(\alpha=0.797)$ had good reliability. In all cases, reliability coefficients were higher at study exit, ranging from $\alpha=0.692$ for suicidal thoughts to $\alpha=0.924$ for helplessness; such values for the propensity and total scale scores were $\alpha=0.915$ and $\alpha=0.912$, respectively. Mean symptom levels declined in all cases from study exit compared to study baseline, as would be expected in a study with an antidepressant treatment arm. 
Table 1. Sample characteristics and descriptive statistics.

\begin{tabular}{|c|c|c|}
\hline Variable & $n(\%)$ & $M \pm S D$ \\
\hline \multicolumn{3}{|l|}{ Gender } \\
\hline Female & $185(66.3 \%)$ & \\
\hline Male & $94(33.7 \%)$ & \\
\hline Age & & $37.24 \pm 13.27$ \\
\hline Hispanic & $49(17.6 \%)$ & \\
\hline \multicolumn{3}{|l|}{ Race } \\
\hline White & $184(65.9 \%)$ & \\
\hline Black or African American & $55(19.7 \%)$ & \\
\hline Asian & $18(6.5 \%)$ & \\
\hline American Indian or Alaska & $1(0.4 \%)$ & \\
\hline \multicolumn{3}{|l|}{ Native } \\
\hline Other & $21(7.5 \%)$ & \\
\hline \multicolumn{3}{|l|}{ Marital status } \\
\hline Single & $164(58.8 \%)$ & \\
\hline Married & $57(20.4 \%)$ & \\
\hline Divorced & $45(16.1 \%)$ & \\
\hline Separated & $8(2.9 \%)$ & \\
\hline Widow & $2(0.7 \%)$ & \\
\hline Partnered & $1(0.4 \%)$ & \\
\hline $\begin{array}{l}\text { Number of people in } \\
\text { household }\end{array}$ & & $2.45 \pm 1.75$ \\
\hline $\begin{array}{l}\text { Number of people in } \\
\text { household, median }\end{array}$ & & 2 \\
\hline \multicolumn{3}{|l|}{ Employment } \\
\hline Employed full time & $88(31.9 \%)$ & \\
\hline Employed part time & $66(23.9 \%)$ & \\
\hline Not employed & $116(42.0 \%)$ & \\
\hline$N / A$ & $6(2.2 \%)$ & \\
\hline Years of education & & $15.08 \pm 2.63$ \\
\hline \multicolumn{3}{|l|}{ Educational attainment } \\
\hline Completed higher education & $122(44.2 \%)$ & \\
\hline $\begin{array}{l}\text { Completed some higher } \\
\text { education }\end{array}$ & $96(34.8 \%)$ & \\
\hline No higher education & $15(5.4 \%)$ & \\
\hline High school diploma & $25(9.1 \%)$ & \\
\hline $\begin{array}{l}\text { General educational devel- } \\
\text { opment (GED) or equivalent }\end{array}$ & $9(3.3 \%)$ & \\
\hline Did not graduate high school & $6(2.2 \%)$ & \\
\hline$N / A$ & $3(1.1 \%)$ & \\
\hline Monthly income & & US $\$ 4831.42 \pm 16,659.92$ \\
\hline Monthly income, median & & US $\$ 2000.00$ \\
\hline \multicolumn{3}{|l|}{ CHRT scores } \\
\hline CHRT Total score & & $25.45 \pm 7.37$ \\
\hline CHRT Propensity score & & $20.02 \pm 6.28$ \\
\hline CHRT Pessimism score & & $4.81 \pm 1.94$ \\
\hline CHRT Helplessness score & & $5.17 \pm 1.79$ \\
\hline CHRT Social support score & & $3.64 \pm 2.14$ \\
\hline CHRT Despair score & & $6.40 \pm 2.82$ \\
\hline CHRT Suicidal thoughts score & & $5.44 \pm 2.33$ \\
\hline HAM-D-17 score & & $18.53 \pm 4.41$ \\
\hline HAM-D Suicide item score & & $0.84 \pm 0.991$ \\
\hline QIDS SR16 Total score & & $18.14 \pm 2.78$ \\
\hline $\begin{array}{l}\text { QIDS SR16 - Thoughts of } \\
\text { death or suicide item }\end{array}$ & & $0.98 \pm 0.83$ \\
\hline
\end{tabular}

CHRT: Concise Health Risk Tracking; HAM- ${ }_{17}$ : Hamilton Depression Rating Scale; QIDS-SR ${ }_{16}$-C: Quick Inventory of Depressive Symptoms-Clinician Rated.

\section{Construct validity}

CHRT-SR 12 factor and total scores were significantly correlated with $\mathrm{HAM}-\mathrm{D}_{17}$ and QIDS-SR ${ }_{16}$ total scores (Table 4). The correlations between the HAM-D ${ }_{17}$ total and CHRT-SR 12 factor scores were modest but statistically significant - with $\rho$ ranging from $0.101-0.205$. Only the CHRT-SR ${ }_{12}$ despair factor was not correlated at the $p<0.05$ level. Correlations between the total QIDS-SR ${ }_{16}$ and CHRT-SR ${ }_{12}$ scores were all positive and statistically significant $(\rho=0.220-0.408$, all $p<0.001$ ), ranging from weak to moderate. In all cases, increased CHRT-SR ${ }_{12}$ factor and total scores were associated with increased HAM-D ${ }_{17}$ and QIDS-SR ${ }_{16}$ total scores, as would be expected.

CHRT-SR $_{12}$ total and Propensity scores were moderately but significantly related $(\rho=0.359-0.442$, each $p<0.001)$ with single items from $\mathrm{HAM}-\mathrm{D}_{17}$ and QIDS-SR ${ }_{16}$ specifically addressing suicide and thoughts of death. All CHRT-SR ${ }_{12}$ factor and total scores were significantly correlated with the QIDS-SR ${ }_{16}$ suicidal ideation item. Scores from the CHRT-SR ${ }_{12}$ suicidal thoughts factor were not significantly correlated with the single suicide item from the HAM-D ${ }_{17}(\rho=0.063, p=0.297)$.

\section{Comparing clinician rated baseline suicidality and CHRT assessments}

Changes in CHRT-SR 12 scores (propensity, suicidal thoughts, and total) for the three levels (none, one, or two/three) of clinicianrated suicidality at baseline were evaluated using mixed model analyses. The models included effects for time, group, and the time $\times$ group interaction. In the analyses there were significant main effects for time, such that scores decreased throughout study weeks: propensity factor score $(F(6,1415)=36.71$, $p<0.0001)$, suicidal thoughts factor score $(F(6,1415)=17.03$, $p<0.0001)$, and total score $(F(6,1415)=38.27, p<0.0001)$. There was also a main effect for baseline suicidality group, such that greater baseline suicidality was associated with higher CHRT-SR $_{12}$ scores: propensity factor score $(F(2,276)=32.65$, $p<0.0001)$, suicidal thoughts factor score $(F(2,276)=3.27$, $p<0.04)$, and total score $(F(2,276)=25.12, p<0.0001)$. No significant interaction between time and baseline suicidality level were found for propensity factor score $(F(12,1415)=0.43$, $p<0.96)$, suicidal thoughts factor score $(F(12,1415)=0.56$, $p<0.88)$, nor the total score $(F(12,1415)=0.40, p<0.97)$. All three clinician-rated groups showed a consistent decrease in scores over the course of treatment, but the difference between groups remained constant across the eight weeks of the acute phase of treatment (Figure 2).

\section{Comparing changes in clinician-rated suicidality and CHRT assessments}

Changes in CHRT-SR ${ }_{12}$ scores (propensity, suicidal thoughts and total) and changes in clinician-rated level (decreased, no change, or increased) between baseline and exit were next evaluated using a mixed model analysis. The models included main effects for time and group and the time $\times$ group interaction. In the analyses there were significant main effects for time, such that scores decreased over time: Propensity factor score $(F(6,1415)=18.25$, $p<0.0001)$, suicidal thoughts factor score $(F(6,1415)=8.23$, $p<0.0001)$, and total score $(F(6,1415)=19.49, p<0.0001)$. There 


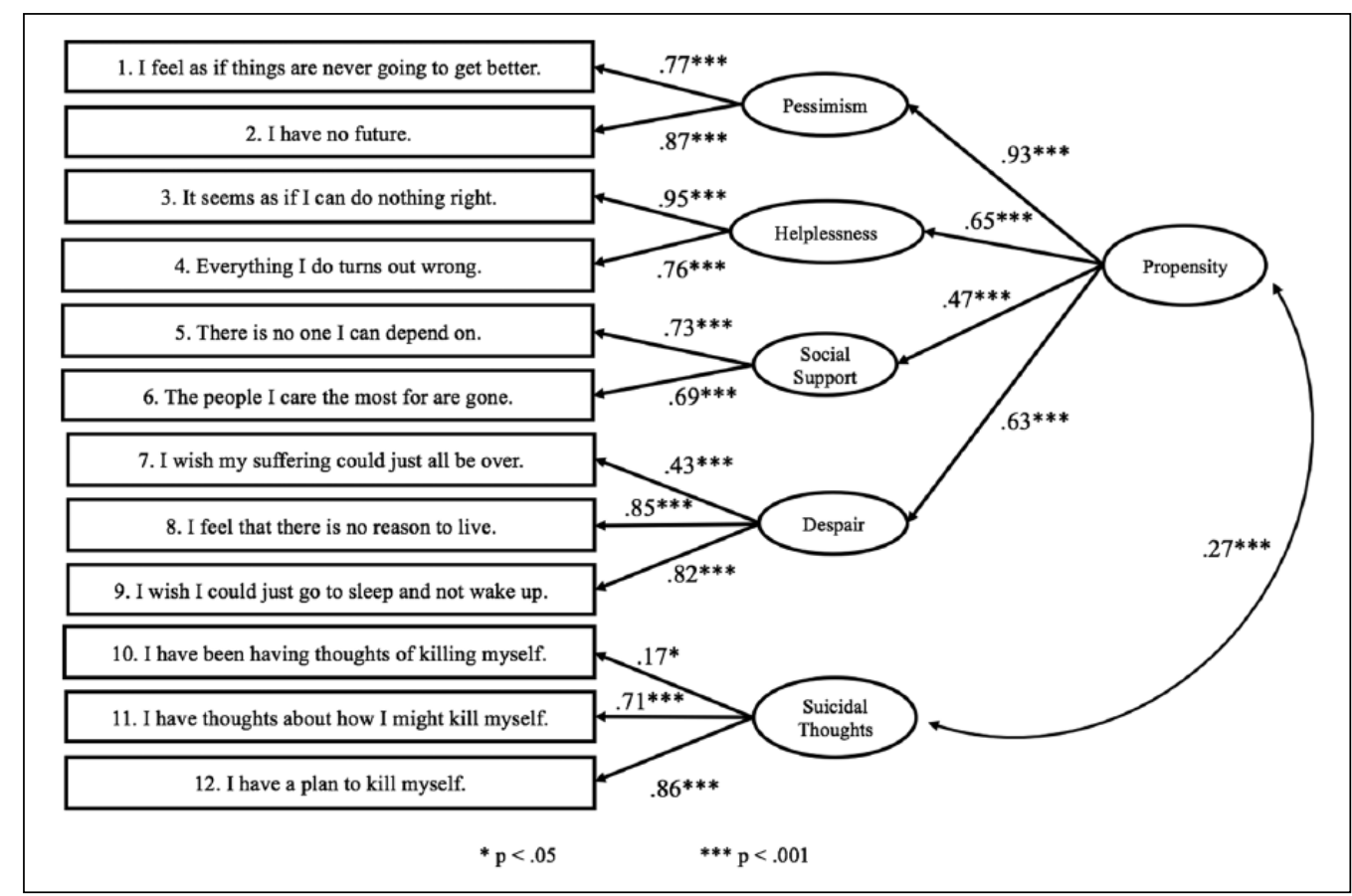

Figure 1. 12-Item Concise Health Risk Tracking-Self Report (CHRT-SR ${ }_{12}$ ) factor model and confirmatory factor analysis (CFA) results.

Table 2. Inter-factor correlations for Concise Health Risk Tracking-Self Report (CHRT-SR ${ }_{12}$ ) scale ( $\rho$, Spearman's rho rank order coefficient test, $n=278)$. $^{\text {a }}$

\begin{tabular}{|c|c|c|c|c|c|c|c|}
\hline $\begin{array}{l}\text { CHRT-SR }_{12} \text { total, factors, } \\
\text { and item scores }\end{array}$ & Total & Propensity & Pessimism & Helplessness & $\begin{array}{l}\text { Social sup- } \\
\text { port }\end{array}$ & Despair & Suicidal thoughts \\
\hline Total & 1.00 & - & - & - & - & - & - \\
\hline Propensity & $0.944^{*}$ & 1.00 & - & - & - & - & - \\
\hline Pessimism & $0.684^{*}$ & $0.732^{*}$ & 1.00 & - & - & - & - \\
\hline Helplessness & $0.638^{*}$ & $0.647^{*}$ & $0.450^{*}$ & 1.00 & - & - & - \\
\hline Social support & $0.577^{*}$ & $0.608^{*}$ & $0.247^{*}$ & $0.278^{*}$ & 1.00 & - & - \\
\hline Despair & $0.722^{*}$ & $0.772^{*}$ & $0.455^{*}$ & $0.302^{*}$ & $0.271^{*}$ & 1.00 & \\
\hline Suicidal thoughts & $0.586^{*}$ & $0.311^{*}$ & $0.217^{*}$ & $0.281^{*}$ & $0.166^{* *}$ & $0.220^{*}$ & 1.00 \\
\hline Item 1 & $0.608^{*}$ & $0.650^{*}$ & $0.904^{*}$ & - & - & - & - \\
\hline Item 2 & $0.650^{*}$ & $0.692^{*}$ & $0.913^{*}$ & - & - & - & - \\
\hline Item 3 & $0.610^{*}$ & $0.638^{*}$ & - & $0.899 *$ & - & - & - \\
\hline Item 4 & $0.588^{*}$ & $0.578^{*}$ & - & $0.934^{*}$ & - & - & - \\
\hline Item 5 & $0.530^{*}$ & $0.543^{*}$ & - & - & $0.885^{*}$ & - & - \\
\hline Item 6 & $0.490^{*}$ & $0.525^{*}$ & - & - & $0.837^{*}$ & - & - \\
\hline Item 7 & $0.437^{*}$ & $0.453^{*}$ & - & - & - & $0.622^{*}$ & - \\
\hline Item 8 & $0.630^{*}$ & $0.682^{*}$ & - & - & - & $0.848^{*}$ & - \\
\hline Item 9 & $0.636^{*}$ & $0.680^{*}$ & - & - & - & $0.883^{*}$ & - \\
\hline Item 10 & $0.438^{*}$ & - & - & - & - & - & $0.448^{*}$ \\
\hline Item 11 & $0.408^{*}$ & - & - & - & - & - & $0.803^{*}$ \\
\hline Item 12 & $0.441^{*}$ & - & - & - & - & - & $0.876^{*}$ \\
\hline
\end{tabular}

aSpearman's rho rank order coefficients were not provided when redundant or when the items did not belong to a factor of the CHRT; ${ }^{*} p<0.001 ;{ }^{* *} p<0.01$.

was also a main effect for suicidality change group, such that scores were lowest in the "no change" group, higher in the "decreased" group, and highest in the "increased" group: propensity factor score $(F(2,276)=12.84, p<0.0001)$, suicidal thoughts factor score $(F(2,276)=5.38, \quad p<0.006)$, and total score $(F(2,276)=12.26, p<0.0001)$.

Importantly, a significant interaction between time and clinician-rated suicidality level change was found for the propensity 
Table 3. Concise Health Risk Tracking-Self Report $\left(\mathrm{CHRT}_{-} \mathrm{SR}_{12}\right)$ measure test-retest reliability and internal consistency reliability, $n=278$.

\begin{tabular}{|c|c|c|c|c|}
\hline \multirow[t]{2}{*}{ Measure } & \multicolumn{2}{|l|}{ Baseline } & \multicolumn{2}{|l|}{ Exit } \\
\hline & $M \pm S D$ & Cronbach's $\alpha$ & $M \pm S D$ & Cronbach's $\alpha$ \\
\hline CHRT-SR $_{12}$ Total (range $0-48$ ) & $25.45 \pm 7.37$ & 0.797 & $16.77 \pm 9.58$ & 0.912 \\
\hline CHRT-SR $_{12}$ Propensity (range $0-36$ ) & $20.02 \pm 6.28$ & 0.803 & $13.05 \pm 8.00$ & 0.915 \\
\hline CHRT-SR $_{12}$ Pessimism (range 0-8) & $4.81 \pm 1.94$ & 0.802 & $3.06 \pm 2.17$ & 0.912 \\
\hline $\mathrm{CHRT}_{-\mathrm{SR}_{12}}$ Helplessness (range $0-8$ ) & $5.17 \pm 1.79$ & 0.835 & $3.17 \pm 2.19$ & 0.924 \\
\hline CHRT-SR ${ }_{12}$ Social support (range $0-8$ ) & $3.64 \pm 2.14$ & 0.670 & $2.51 \pm 2.07$ & 0.785 \\
\hline CHRT-SR $_{12}$ Despair (range 0-12) & $6.40 \pm 2.82$ & 0.728 & $4.32 \pm 3.04$ & 0.824 \\
\hline CHRT-SR ${ }_{12}$ Suicidal thoughts (range $0-12$ ) & $5.44 \pm 2.33$ & 0.570 & $3.72 \pm 2.43$ & 0.692 \\
\hline
\end{tabular}

M: mean; SD: standard deviation.

Table 4. Construct validity for Concise Health Risk Tracking-Self Report (CHRT-SR $\left.{ }_{12}\right)$ scale $(\rho$, Spearman's rho rank order coefficient test, $n=278)$.

\begin{tabular}{|c|c|c|c|c|}
\hline Measure & HAM- $\mathrm{D}_{17}$ total & $\mathrm{HAM}-\mathrm{D}_{17}$ suicide item & $\mathrm{QIDS} \mathrm{SR}_{16}-\mathrm{SR}$ total & $\begin{array}{l}\text { QIDS-SR }_{16}-\mathrm{SR} \text { thoughts } \\
\text { of death or suicide item }\end{array}$ \\
\hline CHRT-SR $_{12}$ Total & $0.201^{*}$ & $0.359 * *$ & $0.408^{* *}$ & $0.433^{* *}$ \\
\hline CHRT-SR $_{12}$ Propensity & $0.191^{*}$ & $0.407^{* *}$ & $0.393^{* *}$ & $0.442^{* *}$ \\
\hline CHRT-SR 12 Pessimism & $0.129 * * *$ & $0.273^{* *}$ & $0.312^{* *}$ & $0.294^{* *}$ \\
\hline CHRT-SR $_{12}$ Helplessness & $0.124^{* * *}$ & 0.104 & $0.344^{* *}$ & $0.140 * * *$ \\
\hline CHRT-SR $_{12}$ Social support & $0.205^{\star}$ & $0.120^{* * *}$ & $0.236^{* *}$ & $0.144^{* * *}$ \\
\hline CHRT-SR ${ }_{12}$ Despair & $0.101^{* * * *}$ & $0.575^{* *}$ & $0.266^{* *}$ & $0.574^{* *}$ \\
\hline CHRT-SR $_{12}$ Suicidal thoughts & $0.127^{* * *}$ & 0.063 & $0.220^{* *}$ & $0.178^{*}$ \\
\hline
\end{tabular}

HAM-D ${ }_{17}$ : Hamilton Depression Rating Scale; QIDS-SR $_{16}-\mathrm{C}$ : Quick Inventory of Depressive Symptoms-Clinician Rated.

${ }^{*} p<0.01 ;{ }^{* *} p<0.001 ;{ }^{* * *} p<0.05 ;{ }^{* * * *} p<0.10$.

factor score $(F(12,1415)=3.48, p<0.0001)$, and total score $(F(12,1415)=3.13, p<0.0002)$, but not for the suicidal thoughts factor $(F(12,1415)=1.18, p<0.30)$. The interaction qualifies the main effects; thus, the pattern of change in scores across time differed by suicidality change group when evaluating the propensity factor score and the total score. The "decreased" and "no change" groups showed a consistent decrease over the course of treatment, while the "increased" group showed an initial decrease in scores that rebounded (i.e. increased) at the end. While visually, the same pattern was observed in the suicidal thoughts score, the interaction was not significant. Taken together, although there was a general decrease in symptoms over time for all three groups combined, the decrease across time only occurred for the "decreased" and "no change" groups when evaluating the total score and the propensity factor as outcomes (Figure 3).

\section{Discussion}

Replicating and extending prior research on the CHRT-SR 12 scale in a placebo-controlled randomized clinical trial for patients with recurrent or chronic major depressive disorder or dysthymia with first onset before age 30 years, our results found a similar factor structure as in prior literature. We specified two factors of propensity and suicidal thoughts, with the propensity factor being composed of four specific first-order factors: pessimism, helplessness, social support, and despair. Reliability and validity were well-established. However, this sample had low rates of suicidal thinking, likely due to the placebo-controlled randomized double-blind nature of the trial. Perhaps as a consequence, poorer model fit, reliability, and item loading for the suicidal thoughts factor as compared to the propensity factor and total score was observed. Furthermore, the suicidal thoughts factor was uncorrelated at baseline with a clinician-rated single item of suicidal thoughts from the HAM-D $\mathrm{D}_{17}$; however, the suicidal thoughts factor was significantly correlated with the single item from the QIDS-SR $_{16}$ - although this correlation was weaker when compared to the total score - highlighting the value of self-ratings for suicidal thoughts.

Indeed, prior research has demonstrated discordance between self-report and clinician-rated assessment of suicidality (Yigletu et al., 2004), with recent suicidal ideation more commonly endorsed on self-report measures (Kaplan et al., 1994). Differential distribution of the item responses in each scale may also explain these divergent results between intercorrelations between the suicidal thoughts factor and the single-item QIDS-SR ${ }_{16}$ and HAM-D ${ }_{17}$, as many more participants endorsed life being empty/not worth living on the self-report QIDS-SR ${ }_{16}$ as compared to the clinician-rated HAM-D ${ }_{17}$. Furthermore, additional differences between the levels on these two items may also impact results and partially explain a significant result for the QIDS-SR ${ }_{16}$ correlation but not for the HAM-D ${ }_{17}$; for example, individuals who make a suicide attempt are automatically rated at the highest level (four on HAM-D ${ }_{17}$ and three on QIDS-SR ${ }_{16}$ ) almost irrespective of the frequency or intensity of suicidal ideation, while suicidal plans are also automatically at the highest level on QIDS-SR ${ }_{16}$ (three) but at a lower level (three) on the HAM-D ${ }_{17}$. Both scales therefore introduce a binary nature of suicide attempts/no attempts that can at times divorce suicidal behavioral from ideation, while the same level of ideation can be 


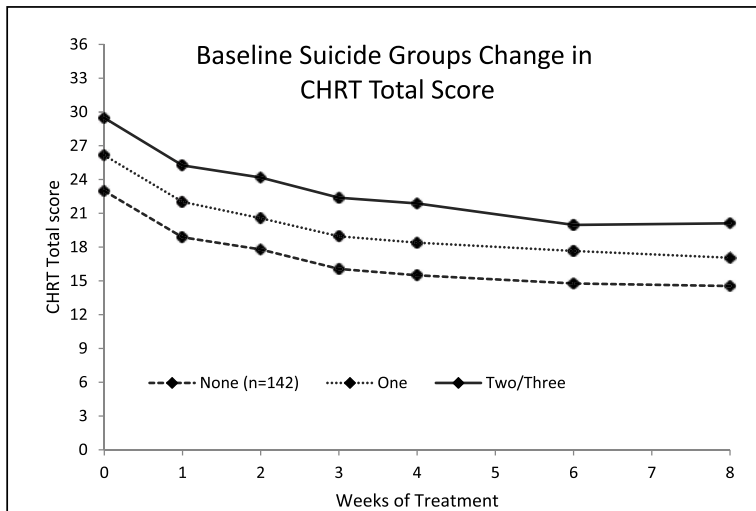

(a)

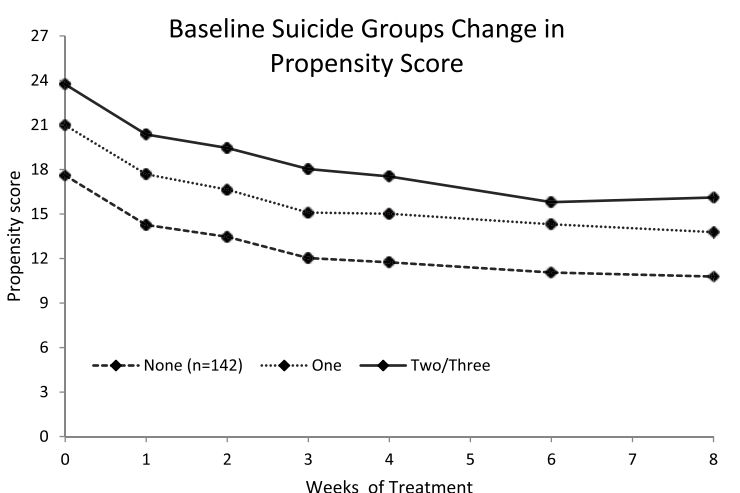

(b)

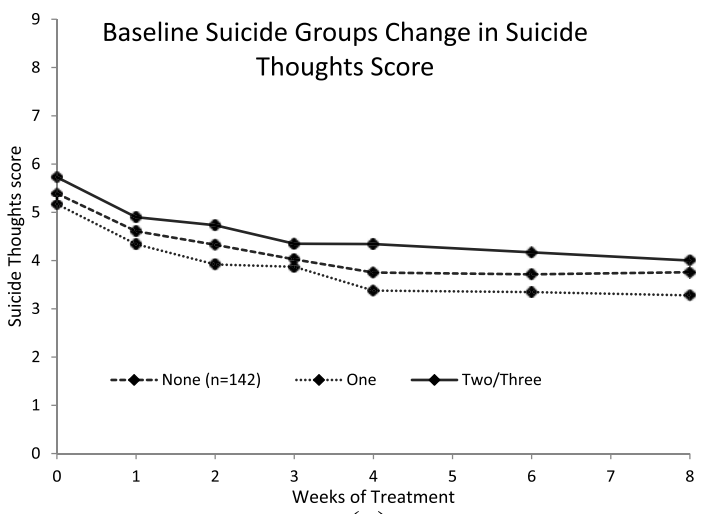

(c)

Figure 2. Change over time for Concise Health Risk Tracking (CHRT) total score (a) propensity factor (b) and suicidal thoughts factor (c) based upon baseline groupings of 17-item Hamilton Rating Scale for Depression (HAM- $\left.D_{17}\right)$ suicidal ideation.

rated slightly differently between measures, due to the different response options. One additional critical aspect is that the study criteria explicitly excluded participants who were "currently actively suicidal or considered a high suicide risk;" this exclusion was necessary for study safety but likely impacted generalizability of results, leading to a restriction of range and limited variability in baseline levels of suicidal thinking.

Extending prior research (de la Garza et al., 2018), we found, as expected, that scores on the total scale and both factors declined over time for all groups defined at baseline by their response to the HAM-D ${ }_{17}$ suicide item, although symptom decline was relatively modest. There was, however, a time by suicidality change group interaction, such that the decrease in symptoms occurred for the total score and the propensity factor in only the "decreased" and "no change" single-item clinicianrated groups. The CHRT-SR ${ }_{12}$ is therefore sensitive to decreases in suicidality over the course of treatment. This study did not demonstrate that the CHRT-SR ${ }_{12}$ was sensitive to increases in suicidal ideation. This is possibly due either to the low frequency of individuals reporting an increase in suicidal ideation during the study (possibly due to antidepressant effects) or the generally low levels of suicidality in this sample. Extending a psychometric evaluation of the CHRT-SR ${ }_{12}$ to samples where substantial increases in suicidality may occur will represent an important direction for future research.

It is also noteworthy that a time $\times$ group interaction focused on changes in CHRT-SR ${ }_{12}$ scores occurred only for the total score factor and propensity factor but not the suicidal thoughts factor. As mentioned above, the limited variability of suicidal thoughts at baseline may have affected these results, since individuals at high risk of future suicidal ideation were excluded. Furthermore, it is important to note that the group with increasing suicidal thoughts as measured by clinicians was comprised of only 19 participants, as opposed to over 100 participants in the other groupings. It is clear that additional research with a larger sample of individuals whose scores paradoxically increase throughout treatment is necessary to better understand the characteristics of this group and to further evaluate the sensitivity of the CHRT-SR 12 in detecting increases in suicidal ideation over time.

There are some important study strengths: the design of a randomized, multi-site controlled trial yields impressive internal validity with both a placebo and a treatment group, and external validity analyses were maximized by multiple intercorrelations with two single-item assessments of suicidal ideation and total scores. The large sample size means that analyses were adequately powered, with the possible exception of the $n=19$ cell for increased CHRT-SR 12 scores. Finally, the inclusion of analyses modeling sensitivity to change in suicidal thoughts over time represents a methodological improvement in contrast to research that examines ideation at only one point in time.

Study limitations include a small proportion of patients with increased suicidal thoughts over time, perhaps because of inclusion criteria that limited variability in suicidality, at least at baseline, and because of the study design that included a treatment arm, whereby 


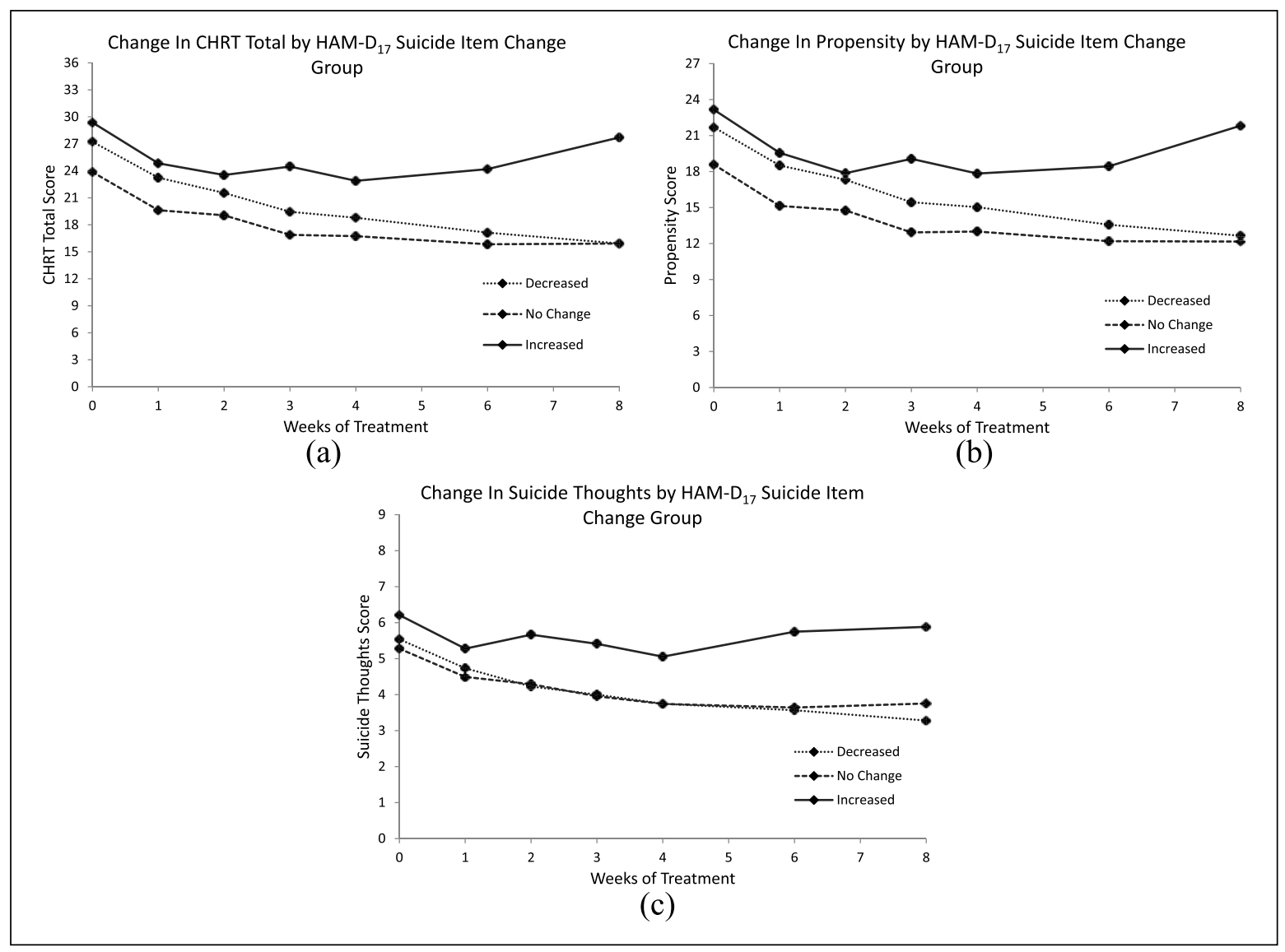

Figure 3. Change over time for Concise Health Risk Tracking (CHRT) total score (a) propensity factor (b) and suicide thoughts factor (c) based upon suicide change groupings of 17 -item Hamilton Rating Scale for Depression (HAM- $D_{17}$ ) suicidal ideation between baseline and exit.

suicidal ideation (alongside other depressive symptoms) would be expected to decrease throughout treatment. Baseline levels of suicidality were also quite low. Future research involving greater numbers of participants with more intense suicidal ideation, or longitudinal increases in suicidal ideation, will be helpful to confirm the utility of the CHRT-SR ${ }_{12}$ in these populations.

In conclusion, we confirm the reliability and validity of the CHRT-SR ${ }_{12}$ in a sample of depressed outpatients participating in a double-blind placebo-controlled trial. The measure and its two subscales were sensitive to decreases in suicidal ideation and related symptoms over time. The CHRT-SR 12 has acceptable psychometric properties for use in efficacy trials, and our results suggest that the reliable, valid, and parsimonious CHRT-SR 12 is a viable alternative to the C-SSRS and can be used in clinical trials, including placebo-controlled antidepressant trials.

\section{Acknowledgements}

The authors wish to acknowledge Jennifer Furman and Jeremy Kee for their administrative support.

\section{Declaration of conflicting interests}

The author(s) declared the following potential conflicts of interest with respect to the research, authorship, and/or publication of this article: $\mathrm{J}$ Trombello currently owns stock in Merck and Gilead Sciences and within the past 36 months previously owned stock in Johnson and Johnson. A Rush has received consulting fees from Akili, Brain Resource Inc., Compass Inc., Curbstone Consultant LLC, Emmes Corp, Holmusk,
Inc., Liva-Nova, Sunovion, Takeda USA, Taj Medical; speaking fees from Liva-Nova; royalties from Guilford Press and the University of Texas Southwestern Medical Center, Dallas, Texas (for the Inventory of Depressive Symptoms and its derivatives). He is also named co-inventor on two patents: US Patent No. 7,795,033: Methods to Predict the Outcome of Treatment with Antidepressant Medication, Inventors: McMahon FJ, Laje G, Manji H, Rush AJ, Paddock S, Wilson AS and US Patent No. 7,906,283: Methods to Identify Patients at Risk of Developing Adverse Events During Treatment with Antidepressant Medication, Inventors: McMahon FJ, Laje G, Manji H, Rush AJ, Paddock S. P McGrath has received funding from the National Institute of Mental Health, New York State Department of Mental Hygiene, Research Foundation for Mental Hygiene (New York State), Forest Research Laboratories, Sunovion Pharmaceuticals, and Naurex Pharmaceuticals (now Allergan). M Oquendo receives royalties for the commercial use of the Columbia Suicide Severity Rating Scale. Her family owns stock in Bristol Myers Squibb. M Weissman has received funding from the National Institute of Mental Health (NIMH), the National Institute on Drug Abuse (NIDA), the National Alliance for Research on Schizophrenia and Depression (NARSAD), the Sackler Foundation, the Templeton Foundation; and receives royalties from the Oxford University Press, Perseus Press, the American Psychiatric Association Press, and MultiHealth Systems. T Carmody has received an honorarium from UT San Antonio. M Trivedi has received consulting fees from or has served on the advisory boards of Alkeremes Inc., Akili Interactive, Navitor, and Otsuka America Pharmaceutical Inc.; holds author agreements with Janssen Asia Pacific and Oxford University Press; honoraria from the American Psychiatric Association; and grants from the Cancer Prevention and Research Institute of Texas (CPRIT), NIMH, NIDA, National Center for Advancing Translational Sciences (NCATS), Johnson and Johnson, 
PCORI. P Adams, T Mayes, R Parsey, M McInnis, M Jha, A Ali, M Killian, and B Grannemann have no conflicts to report. All authors have approved this manuscript.

\section{Funding}

The author(s) disclosed receipt of the following financial support for the research, authorship, and/or publication of this article: The EMBARC study was supported by the National Institute of Mental Health of the National Institutes of Health under award numbers U01MH092221 (MH Trivedi) and U01MH092250 (PJ McGrath, RV Parsey, MM Weissman). The content is solely the responsibility of the authors and does not necessarily represent the official views of the National Institutes of Health. Valeant Pharmaceuticals donated the Wellbutrin XL used in the study. This work was supported by the EMBARC National Coordinating Center at UT Southwestern Medical Center, MH Trivedi, Coordinating PI, and the Data Center at Columbia and Stony Brook Universities. Clinical trials: Establishing Moderators and Biosignatures of Antidepressant Response for Clinical Care for Depression (EMBARC), https://clinicaltrials.gov/ ct2/show/NCT01407094.

\section{ORCID iDs}

Michael O. Killian (iD) https://orcid.org/0000-0002-2287-9007 Madhukar H. Trivedi iD https://orcid.org/0000-0002-2983-1110

\section{References}

Abramson LY, Alloy LB, Hogan ME, et al. (2000) The hopelessness theory of suicidality. In: Joiner TE and Rudd MD (eds), Suicide Science: Expanding the Boundaries. New York, NY: Kluwer Academic/Plenum Publishers, pp. 17-32.

Abramson LY, Metalsky GI and Alloy LB (1989) Hopelessness depression: A theory-based subtype of depression. Psychol Rev 96: 358-372.

Bollen KA (1989) Structural Equations with Latent Variables. New York, NY: Wiley.

Brown T (2006) Confirmatory Factor Analysis for Applied Research. New York, NY: Guildford Press.

Celano CM, Beale EE, Mastromauro CA, et al. (2017) Psychological interventions to reduce suicidality in high-risk patients with major depression: A randomized controlled trial. Psychol Med 47: 810-821.

Centers for Disease Control and Prevention (2016) Data \& Statistics: Fatal Injury Report for 2016. Atlanta, GA: National Center for Injury Prevention and Control.

de la Garza N, Rush AJ, Killian M, et al. (2018) The Concise Health Risk Tracking Self-Report (CHRT-SR) assessment of suicidality in depressed outpatients: A psychometric evaluation. Depression and Anxiety. Epub ahad of print 29 October 2018. doi: 10.1002/da.22855

Foster KT, Li N, McClure EA, et al. (2016) Gender differences in internalizing symptoms and suicide risk among men and women seeking treatment for cannabis use disorder from late adolescence to middle adulthood. J Subst Abuse Treat 66: 16-22.

Giddens JM, Sheehan KH and Sheehan DV (2014) The ColumbiaSuicide Severity Rating Scale (C-SSRS): Has the "gold standard" become a liability? Innov Clin Neurosci 11: 66-80.

Hamilton M (1960) A rating scale for depression. J Neurol Neurosurg Psychiatry 23: 56-62.

Hu L and Bentler PM (1999) Cutoff criteria for fit indexes in covariance structure analysis: Conventional criteria versus new alternatives. Struct Equ Modeling 6: 1-55.

Iosifescu DV, Neborsky RJ and Valuck RJ (2016) The use of the Psychiatric Electroencephalography Evaluation Registry (PEER) to personalize pharmacotherapy. Neuropsychiatr Dis Treat 12: 2131-2142.

Joiner TE (2005) Why People Die by Suicide. Cambridge, MA: Harvard University Press.
Kaplan ML, Asnis GM, Sanderson WC, et al. (1994) Suicide assessment: Clinical interview vs. self-report. J Clin Psychol 50: 294-298.

Kessler RC, Borges G and Walters EE (1999) Prevalence of and risk factors for lifetime suicide attempts in the National Comorbidity Survey. Arch Gen Psychiatry 56: 617-626.

Kline RB (2016) Principles and Practice of Structural Equation Modeling. 4th ed. New York, NY: Guilford Press.

Kohler-Forsberg O, Madsen T, Behrendt-Moller I, et al. (2017) Trajectories of suicidal ideation over 6 months among 482 outpatients with bipolar disorder. J Affect Disord 223: 146-152.

Mann JJ, Apter A, Bertolote J, et al. (2005) Suicide prevention strategies: A systematic review. JAMA 294: 2064-2074.

Mayes TL, Kennard BD, Killian M, et al. (2018) Psychometric properties of the concise health risk tracking (CHRT) in adolescents with suicidality. Journal of Affective Disorders 235: 45-51.

Morris DW, Trivedi MH, Husain MM, et al. (2010) Indicators of pretreatment suicidal ideation in adults with major depressive disorder. Acta Psychiatrica Scandanavica 121: 480-484.

Nierenberg AA, Montana R, Kinrys G, et al. (2017) L-Methylfolate for Bipolar I depressive episodes: An open trial proof-of-concept registry. J Affect Disord 207: 429-433.

Ostacher MJ, Nierenberg AA, Rabideau D, et al. (2015) A clinical measure of suicidal ideation, suicidal behavior, and associated symptoms in bipolar disorder: Psychometric properties of the Concise Health Risk Tracking Self-Report (CHRT-SR). J Psychiatr Res 71: 126133.

Posner K, Brown GK, Stanley B, et al. (2011) The Columbia-Suicide Severity Rating Scale: Initial validity and internal consistency findings from three multisite studies with adolescents and adults. $\mathrm{Am} \mathrm{J}$ Psychiatry 168: 1266-1277.

Reilly-Harrington NA, Shelton RC, Kamali M, et al. (2016) A tool to predict suicidal ideation and behavior in bipolar disorder: The Concise Health Risk Tracking Self-Report. J Affect Disord 192: 212-218.

Rush AJ, Trivedi MH, Ibrahim HM, et al. (2003) The 16-Item Quick Inventory of Depressive Symptomatology (QIDS), clinician rating (QIDS-C), and self-report (QIDS-SR): A psychometric evaluation in patients with chronic major depression. Biol Psychiatry 54: 573-583.

Sanchez K, Killian MO, Mayes TL, et al. (2018) A psychometric evaluation of the Concise Health Risk Tracking Self-Report (CHRT-SR)- a measure of suicidality-in patients with stimulant use disorder. $J$ Psychiatr Res 102: 65-71.

Trivedi MH, McGrath PJ, Fava M, et al. (2016) Establishing moderators and biosignatures of antidepressant response in clinical care (EMBARC): Rationale and design. $J$ Psychiatr Res 78: 11-23.

Trivedi MH, Wisniewski SR, Morris DW, et al. (2011) Concise Health Risk Tracking scale: A brief self-report and clinician rating of suicidal risk. J Clin Psychiatry 72: 757-764.

Villegas AC, DuBois CM, Celano CM, et al. (2018) A longitudinal investigation of the Concise Health Risk Tracking Self-Report (CHRTSR) in suicidal patients during and after hospitalization. Psychiatry Res 262: 558-565.

Williams JB (1988) A structured interview guide for the Hamilton Depression Rating Scale. Arch Gen Psychiatry 45: 742-747.

World Health Organization (2018) Suicide: Key Facts. Available at: http:// www.who.int/en/news-room/fact-sheets/detail/suicide (accessed 20 August 2018).

Yigletu H, Tucker S, Harris M, et al. (2004) Assessing suicide ideation: Comparing self-report versus clinician report. J Am Psychiatr Nurses Assoc 10: 9-15.

Youngstrom EA, Hameed A, Mitchell MA, et al. (2015) Direct comparision of the psychometric properties of multiple interview and patient-rated assessments of suicidal ideation and behavior in an adult psychiatric inpatient sample. J Clin Psychiatry 76: 1676-1682.

Zisook S, Lesser IM, Lebowitz B, et al. (2011) Effect of antidepressant medication treatment on suicidal ideation and behavior in a randomized trial: An exploratory report from the Combining Medications to Enhance Depression Outcomes Study. J Clin Psychiatry 72: 1322-1332. 